University of Montana

ScholarWorks at University of Montana

7-2017

\title{
Reply to Marques et al. (2017): how to best handle potential detectability bias
}

Richard L. Hutto

University of Montana - Missoula, hutto@mso.umt.edu

Follow this and additional works at: https://scholarworks.umt.edu/biosci_pubs

Part of the Biology Commons

Let us know how access to this document benefits you.

\section{Recommended Citation}

Hutto, R. L. (2017), Reply to Marques et al. (2017): how to best handle potential detectability bias. Ecol Appl, 27: 1699-1702. doi:10.1002/eap.1570

This Letter to the Editor is brought to you for free and open access by the Biological Sciences at ScholarWorks at University of Montana. It has been accepted for inclusion in Biological Sciences Faculty Publications by an authorized administrator of ScholarWorks at University of Montana. For more information, please contact

scholarworks@mso.umt.edu. 
Ecological Applications, 27(5), 2017, pp. 1699-1702

(C) 2017 by the Ecological Society of America

\section{Reply to Marques et al. (2017): how to best handle potential detectability bias}

\section{To the Editor}

Marques et al. (2017) write in response to a communications paper in which I (Hutto 2016) question whether a model-based approach is the only way to deal with the detectability problem inherent in bird survey work. The model-based approach to dealing with potential detectability bias is widely viewed as the operational gold standard, and I welcome the comments from experts in this field. The authors did uncover some errors on my part, which I address below, but they also misrepresented my most important points, so I am glad to have the opportunity to respond here.

Misrepresenting my thesis.-In their very first sentence, Marques et al. (2017:1694) state that I “...challenge the need to account for detectability when interpreting data from point counts." Nothing could be further from the truth. In fact, the entire first paragraph of my paper outlines why the known variation in detectability associated with countless factors (including distance from observer, observer identity, time of day, time of season, year, weather, clothing color, noise, wind, temperature, humidity, rain, etc.) represents a potential problem for scientists wanting to compare bird abundances across points in space or time. I end my first paragraph by noting that "if the value of any potential source of bias is not equal among categories being compared (e.g., among vegetation types) or not controlled after the fact through statistical means, then there is a strong chance that any difference in bird abundance might be due to a difference in detectability and not to a difference in vegetation type or whatever variable constitutes the comparison of interest" (Hutto 2016:1287). My position is misrepresented again in the very next paragraph when they imply that I do not believe "...common sense would require that detectability be considered within the context of the study objectives" (Marques et al. 2017:1694). In fact, I do believe that "...the most important thing a researcher can do is use some common sense up front when designing a bird survey so that he or she is unlikely to be fooled by those potential [detectability] problems" (Hutto 2016:1294). Finally, the last sentence in their paper also misrepresents what I wrote. They conclude that "...it is the responsibility of researchers wishing to ignore detectability to justify their choice" (Marques et al. 2017:1697), but I never suggested anywhere in my paper that researchers ignore the detectability issue. The whole point of my commentary was to suggest that detectability is indeed a potential problem, but that common sense, good research design, and simple distance limits should serve to eliminate the danger of being deceived by a measured difference in bird abundance between two treatment categories that arises when birds are differentially detectable in the places that correspond with alternative treatment categories.

The assumption of reasonably high rates of detectability.The authors agree that one might be able to assume a uniformly high level of detectability under some research designs, but they also argue that such an assumption "...must be reviewed and validated" (Marques et al. 2017:1694). I disagree that any level of detectability can be validated. As outlined at the end of my discussion (p. 1293), and as expressed by Emlen (1971:333), one would need to compare the measured and actual abundances at the same places and times to know the actual level of detectability for any given species. Unfortunately, the actual bird abundance at any one place and time is nearly impossible to determine, and I do not believe that actual abundances can be obtained from a modeling exercise based on unmet assumptions associated with repeat sampling from the same point.

Fixed-distance methods are unspecified-The authors are correct in stating that "in the absence of a formal model, the choice [of a fixed-radius distance] is subjective" (Marques et al. 2017:1694), but the method I recommend using to find a suitable fixed distance for any given species relies on a plot of the actual detection distances recorded, so it is not absent a model. In no way does the fixed-radius methodology involve using an "... .unspecified ad hoc 'model' for incomplete detection as a function of distance," as Marques et al. (2017) claim (p. 1694). I will try to be more specific here by suggesting two approaches that one might use to select a suitable fixed radius. First, one could plot the empirically based detection profile for a given species. I should make it clear here that a "detection profile" is not the same thing as a "detection function." The former is merely an areaadjusted plot of the numbers of detections within each of a series of distance categories. The fact that detection profiles are not flat is partly the result of a distancebased effect on detectability (numbers of detections necessarily drop off with increasing distance from the observer), and partly the result of bird movement (not infrequently, a hump-shaped profile can result from birds moving either toward or away from the observer). 
Because birds move, one must surmise where along the detection profile the net flow of individuals during the count was neither toward nor away from the observer. The selection of that distance may be somewhat subjective, but it requires that the observer have intimate knowledge of the biology of the species in question. In most instances, a distance just beyond the peak (as Emlen [1971] originally suggested) is probably the best choice for a fixed distance. This is precisely what I specified in my paper when I suggested choosing a distance "...between the peak and the inflection point on the detection profile" (p. 1291). Experience suggests that one's estimate of bird abundance is not particularly sensitive to the fixed distance chosen, as long as it falls inside the point where the distance-based decline falls steeply. Because empirical data are used to find the fixed distance used for analysis, the fixed radius is selected (or verified as appropriate) after data collection, so fieldwork still entails recording all bird detections and their corresponding distances. A second approach involves testing whether the shapes of the empirically based distance profiles (histograms) differ significantly among the categories of comparison. If not, then there is no reason to worry about a distance-based detectability bias; if they do differ, then one could use distance-limited subsets of the database to find the largest possible fixed distance that no longer produces significantly different detection profiles. Unfortunately, the latter method requires sample sizes (numbers of independent detections) that are almost always well beyond what most studies generate.

Unmet assumptions required of model-based approaches.The authors repeat some of the known assumptions associated with distance sampling, but they ignore the fact that the most important ones are always violated. Specifically, there is no way to ignore the movement of birds during a count, so the most important assumption one must make to build a reliable model (the snapshot assumption) is necessarily violated. The authors note (Marques et al. 2017:1694) that "...time spent at the point is thus a compromise: time must be sufficient to ensure that all animals at or near the point are detected, but not so much that substantial movement occurs," but they are mistaken if they believe (Marques et al. 2017:1694) that “....animal positions [can be] recorded at a snapshot moment, with time before the snapshot used to locate animals, and time after used to confirm locations." There is no way that birds recorded by technicians at the end of a point count were first detected during a snapshot moment some 10 min earlier. Technicians routinely record new detections throughout a count, and birds also move throughout a count (both into and beyond detection range). Not only do birds move, but as any skilled birdwatcher can tell you, many of them move in response to the observer. This is the most important point I would like to make here: known violations of assumptions inherent in the modeling approach compromise the integrity of the methodology, so the use of a simpler, fixed-distance method that carries fewer assumptions might be a reasonable alternative.

Is the detection rate sufficiently high within the fixed radius that I chose for my woodpecker example?.-The authors challenge my claim that a fixed radius of around $80 \mathrm{~m}$ probably yields meaningful results in my woodpecker study by stating (Marques et al. 2017:1695) that they "...tested this claim by fitting a hazard-rate detection function model to this data set." I disagree that one can "test" the veracity of my claim through such a modeling approach.

As I note in 2. The assumption of reasonably high rates of detectability, one cannot know the actual number of birds present and cannot estimate the numbers of birds present through modeling because of unmet assumptions, so one cannot "test" the idea that detectability was at any particular level. Even more importantly, incomplete detectability is not in and of itself a problem; it is variation in detectability rates among categories of comparison that poses a problem, and Marques et al. (2017) did not show that there was a difference in woodpecker detectability rates among categories of fire severity. One thing I now realize is that I failed to account for area in my "detection profile" before I suggested a fixed radius within which distance effects are likely to be negligible. The point at which the detection rate begins to fall off rapidly would, therefore, be something less than the $80-100 \mathrm{~m}$ that I suggested in my paper. Fortunately, this error does not detract from the main points of my paper.

In their appendix, the authors misrepresent the goal behind use of a fixed distance for analysis. The goal is to find that distance where detectability begins to decline rapidly because of distance effects alone. Perfect detection within the fixed distance is not an assumption associated with the method; one must assume only that the detectability (whatever it is) does not differ among categories of comparison. Therefore, I did not mean to imply that my dataset "...provides evidence that detectability is perfect up to about $80-100$ m." Unfortunately, my erroneous use of "detectability" rather than "numbers of detections" in reference to Emlen's "detection profile" in the introduction of my paper probably contributed to the confusion. As Emlen (1971) noted, the "basal" level of detectability independent of distance effects is expected to be something less than $100 \%$ for any species because distance is not the only factor that affects detectability. By using a fixed distance near where detectability begins to fall rapidly due to distance effects, however, one ought to be able to assume that any distance-based differences in detectability among categories of comparison are negligible. A solid research design can be used to minimize the other potential sources of detectability bias. 
Results vary among practitioners. - One of my criticisms of the modeling approach is that different analysts often arrive at different density estimates when modeling detectability. The authors believe that this criticism is misguided, but decades of observing students and others who employ the modeling approach suggest otherwise. The authors then go on to say (Marques et al. 2017:1694-1698) that it is also possible for different analysts to "... select different fixed radii, potentially generating markedly different conclusions." I would argue, however, that the fixed distance chosen (within reasonable limits around the objectively determined peak in a detection function) has little effect on results and is, therefore, unlikely to lead to "markedly different conclusions."

Distance sampling obscures habitat relationships. - In reference to my criticism that the use of distance-sampling methods can obscure bird-habitat relationships, the authors state that this is a problem of scale and not distance sampling per se. I agree that this is a spatial scale-matching problem. The point I was trying to make, however, was that the modeling approach encourages such spatial mismatches between birds detected and the habitat conditions they require. This is because model fitting encourages researchers to keep all data rather than discard distant observations (where birds frequently occur in vegetation conditions that differ markedly from vegetation conditions near the count point itself).

Occupancy vs. detectability.-Concerning occupancy modeling, and as outlined in my paper (Hutto 2016), I do not agree with Marques et al. 2017 (p. 1696) that occupancy models are useful for "... separating out the biological and sampling processes using point-count data" because, once again, one cannot separate the probability of occupancy from the probability of detection as parameterized through repeated sampling in time or space.

Frequency of occurrence and habitat suitability.-In my paper, I claimed (Hutto 2016:1291) that a point where a bird is frequently detected is likely to be a much more suitable place than a point where a bird is rarely detected. The authors countered by noting that they could imagine this to be utterly wrong, and that assuming so could even be "dangerous" (Marques et al. 2017:1696). I can also imagine the assumption to be wrong, but theoretical possibilities lose a lot of weight when extensive field experience suggests that they are just that: theoretical possibilities. If one could show, as the authors suggest, that animals are more conspicuous in places they avoid, or that singing male birds really prefer to be in places where they are super quiet and inconspicuous, then I concur, the use of naive abundances would be a problem. Until then, common sense suggests that birds are vocally and visually conspicuous in places they defend.

Rigorous science requires density estimates, not indices of abundance.-The authors claim (Marques et al. 2017:1697) that a fixed-distance approach “...may be useful for generating hypotheses to be further investigated using more rigorous approaches," but "...to support the effective conservation and management of wildlife populations, we need reliable estimates of population size." First, to suggest that a fixed-distance approach is inadequate to the task of wildlife management is not only condescending, but also flat-out wrong. Population estimates are necessary only for the narrow questions that require them; they are not required for effective conservation and management. Indeed, many local, state, and federal environmental regulations and mandates have been stimulated by results based on simple indices of abundance. Second, I fail to see how a fixed-radius approach is any less rigorous than a modelbased approach. Rigor has nothing to do with the method chosen; it has everything to do with how meticulous, thorough, and careful one is with whatever method one employs.

In conclusion, I encourage researchers who conduct bird surveys to ask themselves whether model-based approaches are the only (or even the best) way to deal with potential detectability problems. Are the research biologists who use a traditional (fixed-distance) approach to studying bird abundance across space or time routinely deceived? Is there convincing evidence that published conclusions based on fixed-distance surveys are generally off base? I still believe that " ... to this day, there is no evidence that people who design their studies to simultaneously minimize many potential sources of bias and then work with data collected from within a reasonably limited radius have ever been deceived or misled by their results" (Hutto 2016:1293). Marques et al. (2017) believe "... it is unjustified to state that model-based approaches in general, and those that account for detectability in particular, should be avoided altogether." I agree, and I would argue that the same goes for fixed-distance methods. I choose to avoid modeling methods to control for potential distancebased detectability bias because known assumption violations carry what I view to be unacceptable risks. I also believe that a fixed-distance survey method coupled with solid research design is a perfectly satisfactory way to deal with potential distance-based detectability problems, and that the method should not be universally condemned by reviewers and editors the way it currently is. 


\section{Literature Cited}

Emlen, J. T. 1971. Population densities of birds derived from transect counts. Auk 88:332-342.

Hutto, R. L. 2016. Should scientists be required to use a modelbased solution to adjust for possible distance-based detectability bias? Ecological Applications 26:1287-1294.

Marques, T. A., et al. 2017. Model-based approaches to deal with detectability: a comment on Hutto (2016). Ecological Applications 27:1694-1698.
Richard L. Hutto

Division of Biological Sciences, University of Montana,

Missoula, Montana 59812 USA

E-mail:hutto@mso.umt.edu

Manuscript received 2 April 2017; accepted 18 April 2017. Corresponding Editor: Andrew O. Finley. 